\title{
Ethnisierungsprozesse und Grenzen
}

\author{
Concha Maria Höfler und Maria Klessmann
}

\begin{abstract}
Der Beitrag widmet sich dem Verhältnis von Grenzziehungen und Ethnisierungspraktiken und strebt eine systematische Darstellung des Zusammenhangs von Ethnizität und Grenze an. Einerseits kann Ethnizität als Produkt einer (soziosymbolischen) boundary gedacht werden, da sie sich häufig über diese Art von Grenzziehungen konstituiert, andererseits ist der Zusammenhang zwischen Ethnizität und (politisch-territorialen) borders interessant, wenn es z.B. um ethnisch aufgeladene Grenzkonflikte geht oder staatliche Institutionen Ethnizität als Differenzkriterium an der Grenze verwenden.
\end{abstract}

\section{Schlagwörter}

Ethnisierung, Nationalisierung, Grenzziehungen, Grenzforschung, Ethnizitätsforschung

\section{Zum Verhältnis von Grenzziehungen und Ethnisierungspraktiken: eine Einleitung}

Ausgehend von der Beobachtung, dass es an einer systematischen Darstellung des Zusammenhangs von Grenzziehungen und Ethnisierungsprozessen mangelt, möchte der folgende Beitrag diese Lücke füllen und das komplexe Wechselverhältnis zwischen beiden Phänomenen genauer beleuchten. Das Verhältnis von Ethnizität und Grenzen geht sowohl wissenschaftlich als auch im Alltagsverständnis meist im Begriff der ethnischen Grenze auf. Als soziosymbolische Grenzen zwischen Gruppen oder Individuen, die auf Vorstellungen ethnisierter Gemeinsamkeiten bzw. Differenzen beruhen, spielen sie demnach in Identifikations- und Abgrenzungsprozessen eine zentrale Rolle. Bereits Georg Simmel (1908/2013) beschreibt die grundlegende Konstitution von Gesellschaften durch innere Grenzziehungen, die mit den geschaffenen Abgrenzungen gleichzeitig Zugehörigkeiten etablieren (vgl. auch Schroer in diesem Band). Gesellschaft wird demnach durch soziokulturelle Grenzziehungen strukturiert, die über Zugehörigkeiten und Differenzierungen ,entscheiden', wie sie auch im Falle ethnisierter Kollektive oder einzelner Akteur*innen zum Tragen kommen.

Davon ausgehend machen verschiedene wissenschaftliche Perspektiven teilweise implizit, teilweise explizit das Verhältnis von Grenzen und Ethnizität zum Thema ihrer Forschung. Dabei wird der Gegenstand in seiner ganzen Breite abgedeckt und behandelt beispielsweise ,ethnische Konflikte', den Schutz von Minderheiten, soziokulturelle Merkmale, die als Marker von Ethnizität dienen, oder transnationale Gemeinschaften, die über staatliche Grenzen hinweg ihre Zusammengehörigkeit pflegen oder durch veränderte Grenzziehungen (sprachliche) Unterschiede entwickeln (vgl. Barth 1969; Orywal/Hackstein 1993; Brubaker 2002; 2009; Bös 2007; Solleder 2017; Voss 2018). Dabei wird der Ethnizitätsforschung immer wieder eine starke Vermischung alltagsweltlicher Vorstellungen und wissenschaftlicher Betrachtungsweisen sowie ein Mangel an fächerübergreifender Auseinandersetzung vorgeworfen (vgl. Alonso 1994; Brubaker 2002; 2009; Müller/Zifounun 2010). Steht die Grenze als komplexes Phänomen und Differenzierungsmoment im Vordergrund, tut sich auch die sozial- und kulturwissenschaftlich informierte Grenzforschung mit Eindeutigkeit im Hinblick auf Analysegegenstand und analy- 
tische Begriffe nicht immer leicht. Sobald die nationalstaatliche Grenze nicht im Zentrum der Forschung steht oder zumindest am Rande mitbehandelt wird, werden häufig zwei (widerstreitende) Forschungsströmungen postuliert und die Frage gestellt, welchen analytischen Mehrwert ein Grenzbegriff in anderen Kontexten als der Geografie, Geschichte oder Politikwissenschaft mit sich bringt. Gegenübergestellt werden dabei klassische Border Studies und Forschung, die sich mit soziosymbolischen Grenzziehungen beschäftigt (vgl. Newman 2006; Parker/Vaughan-Williams 2012; Gerst et al. 2018a; vgl. auch Brambilla et al. in diesem Band). An dieser Stelle erscheint es uns wichtig, zu betonen, dass sich (national-)staatliche Grenzen nicht losgelöst von soziosymbolischen Grenzen verstehen lassen.

Unser Beitrag zielt darauf ab, Ethnie bzw. Ethnizität als ein Phänomen zu behandeln, welches in Prozessen sozialer Konstruktionen hergestellt und verhandelt wird. Deswegen sprechen wir in diesem Beitrag weitestgehend von Ethnisierung, Ethnisierungsprozessen oder Praktiken der Ethnisierung. Dabei tragen wir auch der rassifizierenden bzw. mindestens biologisierenden Problematik des Begriffs Ethnizität in seiner historischen Kontingenz und Verwendung Rechnung (vgl. Alonso 1994; Bös 2005; Sökefeld 2007). ${ }^{1}$ Ethnizität kann somit als Ergebnis der Herstellung eines (letztlich biologisch essenzialisierten) Anderen analysiert, nicht aber vorausgesetzt werden. Wie Richard Jenkins es für soziale Identitäten generell festhält, geht es darum, diese als "practical accomplishments rather than static form” (Jenkins 1994, S. 218, Herv. i. O.) zu begreifen und zu beschreiben. Dass Prozesse der Ethnisierung dabei in den allermeisten Fällen auch Prozesse des Abwertens bzw. Aufwertens einschließen, muss kritisch mitberücksichtigt werden. Aus intersektionaler Perspektive ließe sich kritisch anmerken, dass wir uns mit dem Fokus auf Ethnisierungsprozesse lediglich einer einzigen sozialen Kategorie widmen. Dass Ethnizität bzw. Ethnisierung nur eine mögliche Form verschiedener Differenzkategorien (und Ungleichheitsdimensionen) darstellt, die (häufig) nicht von anderen, wie Klasse, Geschlecht oder Alter, zu trennen ist, verlieren wir jedoch nicht aus dem Blick, sondern setzen wir voraus (vgl. hooks 1981; Anzaldúa 1987; Winker/Degele 2009).

Im Folgenden wird es darum gehen, die vielschichtigen Beziehungen von Ethnisierungs- und Grenzziehungsprozessen genauer auszubuchstabieren und deren Implikationen für eine Ethnizitätsforschung mit Grenzperspektive sowie Grenzforschung mit Ethnisierungsperspektive darzulegen. Im folgenden Teil 2 gehen wir auf das Wechselverhältnis von soziosymbolischen Grenzen und Ethnisierung näher ein, um daran anknüpfend die politisch-geografische Grenze, wie sie traditionell hauptsächlich in den Border Studies verhandelt wird, im Hinblick auf ihre Berührungspunkte mit Ethnisierungs- und Nationalisierungspraktiken zu beleuchten (Teil 3). Eine Zusammenführung beider Forschungsfelder nehmen wir anhand einer Untersuchung der während der COVID-19-Pandemie gezogenen Grenzen vor (Teil 4).

\section{Ethnizität in der Boundary-Forschung}

Für die folgenden Ausführungen ist die hier angewandte Unterscheidung zwischen boundaries und borders relevant. Wir greifen auf diese im Englischen geläufige Unterscheidung zurück, um die Unterteilung zwischen soziosymbolischen Grenzziehungen (boundaries) sowie politisch-territorialen Demarkationen (borders) kenntlich zu machen (vgl. Lamont/Molnár 2000). Dem liegt ein Verständnis von politisch-territorialer Grenze als ein Fall von Grenzen zugrunde,

1 Siehe auch Debatten um Primordialismus und Konstruktivismus in der Erforschung von Ethnizität, z.B. bei Handler (1985); Spivak (1988); Mahmood (1996); Moya/Hames-Garcia (2000). 
der sich aus dem komplexen Zusammenspiel verschiedener Dimensionen von Grenzziehungen speist (Haselsberger 2014; Gerst et al. 2018b). Um das Wechselverhältnis von boundaries und Ethnisierung zu betrachten, widmen wir uns zunächst der Herstellung ethnisierter Grenzen und folgen hier vor allem sozial- und kulturwissenschaftlichen Arbeiten, die versuchen, das Verhältnis empirisch zu fundieren. Da ethnisierte Grenzziehungen häufig in Interaktionen anhand von Kategorisierungen organisiert und somit beobachtbar werden, kommen wir anschließend vom breiten soziokulturellen Blick auf die eher mikroanalytische Perspektive interaktionaler Grenzziehungsprozesse. Dies wiederum leitet zu analytischen Perspektiven auf die Stabilisierung und Verfestigung ethnisierter Grenzziehungsprozesse über, die wir am Beispiel der Nation verdeutlichen.

\subsection{Praktiken ethnisierter Grenzziehungen}

Im allgemeinen Verständnis gilt Ethnizität häufig als „grundlegende [soziale] Identität“ mit einem bestimmten „Set von kulturellen Ausstattungen und Identifikationen“ (Feischmidt 2007, S. 52), die zumeist als , angeboren` essenzialisiert werden:

„Alltagstheoretisch gilt ethnische Zugehörigkeit als ein askriptives und grundsätzlich unveränderbares Personenmerkmal, dessen Zuweisung mit der Geburt, also qua Natur bzw. (Bluts-)Verwandtschaft erfolgt und sich, wenn überhaupt, nur mit sehr großem Aufwand nachträglich erwerben lässt“ (Müller/Zifonoun 2010, S. 11).

Diese ,grundlegende Identität‘ findet sich auch in gängigen Konzeptionalisierungen der Nation wieder, da „ethnische Differenzen soziale Folgen nach sich ziehen können, wie z.B. politische Vergemeinschaftungen, die Bildung von Nationalstaaten, Diskriminierungen und soziale Exklusion bis hin zur Verfolgung und Auslöschung ethnischer Kollektive“ (ebd.). Demnach ist dieses alltägliche Verständnis von Ethnizität in seinem starken Bezug auf verwandtschaftliche Beziehungen auch biologisierend und mithin letztlich rassifizierend. ${ }^{2}$

Im zugeschriebenen und fixierten Charakter der Ethnizität zeigt sich schon eine Beziehung zwischen Ethnizität und sozialen Grenzen: In der politischen oder kulturellen Vergemeinschaftung über Ethnizität scheinen soziokulturelle Grenzen festgeschrieben und grundsätzlich unveränderbar. Wissenschaftliche Perspektiven betonen dabei jedoch die hohe Dynamik sozialer Grenzen und Ethnizität (vgl. Nail 2016), die nicht nur von ihrer Prozesshaftigkeit zeugt, sondern es auch nahelegt, sich mit den Praktiken ethnisierender Zuschreibungen und deren Aushandlungen zu beschäftigen. Dabei sind beobachtbare Selbst- und Fremdpositionierungen im Sinne ethnisierender Zuschreibungen immer auch als (Grenz-)Aushandlungen von Agency und Macht zu verstehen (vgl. u.a. Römhild 2007; Wimmer 2013).

Die verschiedenen Ansätze, die soziale Grenzen und Ethnisierung zusammenführen, sehen meist in der Praxis der ethnisierenden Humandifferenzierung selbst eine Grenzziehung. Fredrik Barth, der mit seinem Text zu Ethnic Groups and Boundaries (1969) wegweisend für die Analyse von sozialen Grenzziehungen zwischen Gruppen war, wendet sich dezidiert gegen ein primordiales Verständnis von Ethnizität: „[E]thnic groups are categories of ascription and identification by the actors themselves, and thus have the characteristic of organizing interaction between people“ (ebd., S. 10). Barth macht deutlich, dass es nicht ,objektive“ Differenzen

2 Für eine ausführliche Diskussion siehe auch Mathias Bös (2005). 
sind, sondern die Signifikanzen der Akteur*innen, die über Selbst- und Fremdpositionierungen entscheiden und folglich auch über die (sozialen) Grenzziehungen. Damit führt er Grenze als grundlegenden Begriff für die Untersuchung von Ethnisierungsprozessen ein. In seinem späteren Text Boundaries and Connections (2000) beschreibt Barth drei verschiedene Abstraktionsniveaus von boundaries: „[L]iterally, boundaries divide territories ,on the ground“, more abstractly, they set limits that mark social groups off from each other and finally, they provide a template for that which separates distinct categories of the mind" (ebd., S. 17). Wenn er dabei also vom Beobachtbaren zum Abstrakten kommt, macht er deutlich, wie Kategorisierungen und Klassifizierungen maßgeblich über Grenzziehungen organisiert werden. Soziosymbolische oder soziokulturelle Grenzen dienen laut Barth in erster Linie der Unterscheidung bzw. Klassifizierung von Gesellschafts- oder Gruppenmitgliedern anhand von Gender, Alter oder Ethnie wobei hier die Grenzen entlang einzelner Kategorien und nicht entlang komplexer intersektionaler Zugehörigkeiten verlaufen. Er hält weiter fest, dass eine Unterscheidung an sich zunächst keinen (sozialen) Effekt hat oder haben muss: „[M]aking a distinction does not necessarily entail drawing a boundary“ (ebd.). Erst wenn eine Differenzierung Folgen nach sich zieht, wird sie zu einer (sozialen) Grenze gemacht; so z.B., wenn die Unterscheidung entlang der Kleidung festlegt, ob jemand als angemessen gekleidet gilt und eine Veranstaltung besuchen darf oder nicht (vgl. auch Lamont/Molnár 2000). Zeitigt die Differenzierung demnach soziale Auswirkungen, kann sie als eine soziale Praxis des Grenzziehens beschrieben werden. Ethnizität begreift Barth in diesem Zusammenhang als anzunehmendes Kontinuum, welches, wenn es relevant wird, zu etwas Trennendem und Einschließendem, also zu einer Grenzziehung werden kann (Barth 2000, S. 30).

Richard Jenkins rückt in seiner ebenfalls einflussreichen Arbeit Rethinking Ethnicity (1994, 2008) mehr die ,Außenperspektive‘ im Sinne von Fremdzuschreibungen in den Fokus. Er blickt auf institutionalisierte Formen von Ethnisierung als soziale Grenzziehungen, die sich letztlich auch in Individuen, einschreiben'. Dies äußert sich folglich in den Handlungen und Interaktionen jener Personen, die von diesen Zuschreibungen adressiert werden. Praktiken der Ethnisierung sind somit als eine Formation zu denken, die auf (soziale) Grenzen und Akteur*innen gleichermaßen wirken, zur Aktualisierung und (retrospektiven) Legitimation von (sozialen) Grenzen herangezogen werden und auch selbst als Grenze fungieren können. Jenkins macht deutlich, dass Ethnizität, wie andere soziale Zuschreibungen, im Zuge sozialer Interaktion mobilisiert wird. Soziale Zuschreibungspraktiken beschreibt er somit als Akte der Grenzziehung zwischen einem Wir und den Anderen, wobei an derart gezogenen Grenzen auch kulturelle Differenz verhandelt wird: „Because it takes two, ethnicity can only happen at the boundary of ,us' in confrontation or by contrast with ,them'. And as the sense of ,us' changes, so the boundary between ,us' and ,them“ shifts“ (Jenkins 2008, S. 20). Ein analytischer Blick auf Ethnisierungspraktiken und Grenzziehungen erfordert demnach auch eine Sensibilität für die sozialen Folgen dieser Prozesse.

\subsection{Der analytische Blick auf Grenzen und Ethnisierungen}

Vor allem in der nordamerikanisch geprägten Soziologie wurden Verhältnisse von Ethnisierung und soziosymbolischer Grenzziehung thematisiert und in den letzten Jahren durch namhafte Vertreter*innen wie Andreas Wimmer (2008a; 2013) und Rogers Brubaker (2002; 2009) in 
ihrer Dynamik herausgearbeitet. Sie plädieren dafür, den situativen, prozeduralen Charakter von Grenzherstellungen und -(de)stabilisierungen vornehmlich im Bezug zu ,ethnischen Gruppen' in den Fokus zu rücken und diese Prozesse weniger selbstverständlich vorauszusetzen. Rogers Brubaker arbeitet sich dabei maßgeblich an der Verwendung des Begriffs Gruppe ab und macht dies an ,ethnischen Gruppen' deutlich. Mit der Voraussetzung von Gruppen als „things-in-the-world“ finde auch eine Art Überethnisierung sozialer Gegebenheiten statt (,an ,overethnicized" view of the social world“, Brubaker 2004, S. 12) und umgekehrt für die Betrachtung von Ethnizität eine Überbetonung vorausgesetzter Gruppen. ,Ethnische Gruppen‘ wurden demnach häufig als klar abgegrenzte - und abgrenzbare - Entitäten verhandelt, ohne sie als spezifische Konstruktionen in ihrer Situativität, Kontextabhängigkeit und Dynamik genauer zu betrachten. Dies lässt sich z.B. an der medialen Berichterstattung über bewaffnete Konflikte ablesen: Zuallermeist werden Kurd*innen, Palästinenser*innen, Kosovo-Albaner*innen, Tamil"innen als homogene Kollektive mit gemeinsamen Zielen und einer gemeinsamen Herangehensweise dargestellt (vgl. ebd.).

Andreas Wimmer spricht in diesem Zusammenhang von „situationeller Flexibilität“ ethnisierter Vorstellungen (Wimmer 2000, S. 53), da der Glaube an das ,Schicksalhafte', ,Naturgegebene` für große Beständigkeit ethnisierter Kategorien sorge. Er macht sich für eine analytische Grenzziehungsperspektive innerhalb der Migrations- und Ethnizitätsforschung stark, womit einer Naturalisierung, Überbetonung und Vereinfachung bestimmter, eigentlich komplexer Zusammenhänge entgegengewirkt würde. Durch eine „Grenzziehungsperspektive verlagert [sich] unsere Aufmerksamkeit sowohl auf die Aushandlungsprozesse [...], als auch auf die verschiedenen korporativen Akteure“ (Wimmer 2008b, S. 71), womit der Blick auf die Subjektivität und auf die komplexen Akteur*innenkonstellationen verdeutlicht wird. Damit geraten Prozesse der sozialen Kategorisierung, wie beispielsweise der Ethnisierung, als Grenzziehungen und -verschiebungen in den Blick, die ansonsten schnell statisch und essenzialistisch erscheinen.

Geht es beim „ethnic boundary making“ (Wimmer 2008a; 2013) stark um eine auf (kollektive) rationale Strategien gerichtete Perspektive, steht in einer stärker praxeologischen Perspektive (wie z.B. in der Ethnomethodologie), die alltägliche Herstellung sozialer Realitäten im Vordergrund. Analytisch wird die Ethnisierung als eine mögliche Form sozialer Differenzierung somit nicht als Ausgangspunkt oder Gegebenheit vorausgesetzt bzw. vorangenommen (vgl. Bergmann 2010). Vertreter*innen praxeologisch geprägter Forschung postulieren, dass Ethnizität nicht am Anfang der wissenschaftlichen Betrachtung stehen kann, sondern als in ihrem Entstehen, also im Herstellungsprozess zu beobachtendes Phänomen. Ethnizität wird dann als Ressource verstanden, die z.B. in einer Interaktion als Kontextwissen eingebracht und herangezogen werden kann. Ethnizität als Ressource kann auch produktiv für die Akteur*innen gemacht werden, wenn Ethnizität beispielsweise als identitätsstiftendes Moment bewusst herangezogen und damit eine (aktive) Setzung vorgenommen wird. Damit steht die Ethnisierung nicht mehr ausschließlich am Ende eines Herstellungsprozesses, sondern bereits zu Beginn und vor der analytischen Leistung der Ethnograf*innen (Wolff 2006, S. 261; vgl. Wolff/Schönefeld 2011). Eine solche bewusste Vereinnahmung von Ethnizität durch Akteur*innen, die sich als Mitglieder ethnischer Gruppen verstehen, muss demnach auch als Möglichkeit ernst genommen und für eine wissenschaftliche Perspektive produktiv gemacht werden (vgl. Brubaker 2004). In der Gleichzeitigkeit zwischen prozesshafter Herstellung und Stabilität lassen sich Ethnizität und Grenzen perspektivisch zusammendenken: Beide können zu Beginn und am Ende analytischer Arbeiten stehen, durch Praktiken hergestellt werden und gleichzeitig diese 
Praktiken legitimieren. Ethnizität und Grenzen können demnach sowohl emische als auch etische Analysekonzepte sein.

Angelehnt an Rogers Brubaker, der Ethnizität als symbolisches Codierungsphänomen fasst, richtet Matthias Bös den Blick auf die Herstellung und die Akteur*innen dieser Codierungen und Kategorisierungen: „Alle ethnischen Grenzdefinitionen beziehen sich auf von den Mitgliedern sinnhaft geglaubte Grenzen“ (Bös 2007, S. 54, Herv. i. O.). Oder wie bereits Fredrik Barth es verdeutlicht, geht es um die ,Eigenschaften', die für die Akteur"innen selbst bedeutend sind: „The features that are taken into account are not the sum of ,objective differences, but only those which the actors themselves regard as significant" (1969, S. 14). In dieser grenztheoretischen Konzeption von Ethnizität ist demnach zentral, wie und von wem ethnisierte Grenzen gezogen, für wen sie damit ,sinnhaft ${ }^{\star}$ und wirkmächtig werden. In dem Wechselspiel zwischen Sinnhaftigkeit und Wirkmächtigkeit macht sich bereits die Unterscheidung zwischen symbolischen Grenzziehungen und deren sozialer Verhärtung deutlich. Einerseits werden symbolische Grenzen von Akteur*innen hergestellt: „Symbolic boundaries are conceptual distinctions made by social actors to categorize objects, people, practices, and even time and space“ (Lamont/Molnár 2002, S. 168). Andererseits verhärten sich symbolische Grenzen in der Objektivierung als soziale Grenzen. „Social boundaries are objectified forms of social differences manifested in unequal access to and unequal distribution of resources (material and nonmaterial) and social opportunities" (ebd.).

Mit Michèle Lamont und Virág Molnár lässt sich davon ausgehen, dass symbolische Grenzziehungen als konzeptuelle Unterscheidungen auf einer intersubjektiven Ebene der Kategorisierung dienen. Damit sind weitere Implikationen für eine grenztheoretische Perspektive auf Ethnisierungspraktiken gewonnen. Soziale ethnisierte Grenzen verdichten sich im Zusammenspiel zwischen Akteur*innen und Institutionalisierungen:

„[S]ymbolic and social boundaries should be viewed as equally real: The former exist at the intersubjective level whereas the latter manifest themselves as groupings of individuals. At the causal level, symbolic boundaries can be thought of as a necessary but insufficient condition for the existence of social boundaries" (ebd., S. 169).

Athanasios Karafillidis reagiert in seinem Text Grenzen und Relationen (2009, S. 108f.) allerdings kritisch auf diese Unterscheidung und stellt heraus, dass symbolische Grenzziehungen ebenfalls soziale Grenzziehungen sind. Daher schlägt er eine Differenzierung in soziale „Grenzziehung und ihrer Institutionalisierung“ (ebd., S. 109) vor. Eine institutionalisierte Grenze ist somit zunächst eine, die soziale Differenzierungen über die intersubjektive Ebene hinaus festschreibt, beispielsweise in ethnisierenden Stereotypen, die zu regelmäßiger Ungleichbehandlung aufgrund dieser Stereotype führen können.

Wie bereits deutlich geworden ist, bringen soziale Differenzierungen nicht zwingend soziale Grenzziehungen mit sich, diese Verfestigungen sind vielmehr kontextabhängig - und lassen sich auch wieder ,lösen ${ }^{63}$. Wenn es jedoch zu Verfestigungen in Form ethnisierter Grenzziehungen kommt, gilt es, die Beziehung zwischen Kategorisierungen, Grenzziehungen und Ethnisierung als „ongoing interactional accomplishment“ (West/Fenstermaker 1995, S. 9) und Stabilisierungsprozesse zu begreifen.

3 Ein besonders eindrückliches Beispiel ist die zu Beginn des letzten Jahrhunderts erfolgte Kategorisierung von italienischen und jüdischen Einwanderer*innen in den USA als ,Schwarz', die damit in der sozialen Hierarchie Afroamerikaner*innen gleichgestellt und entsprechend (schlecht) behandelt wurden (vgl. Wimmer 2008a). 


\subsection{Zur Stabilisierung ethnisierter Grenzziehungen}

Ausgehend von der Annahme, „dass alle soziale Differenzierung praktiziert werden muss, also Teil einer Vollzugswirklichkeit ist, wobei Individuen weder als Akteure noch als Träger von Identitäten, sondern als bloße Vermittler sozialer Praxis betrachtet werden " (Hirschauer 2014, S. 182, Herv. i. O.), wird in der Forschung die soziale Praxis der Ethnizitätsherstellung und Stabilisierung in den Blick genommen. Die Praxis der Ethnisierung wird dann „als eine [...] praktische Leistung betrachtet, als etwas, das ,geschieht', wenn ethnische Kategorien im Lauf einer bestimmten interaktionalen Bewegung für die Beteiligten relevant werden “ (Brubaker 2007, S. 103). Bereits die Selbst- oder Fremdzuschreibung einer als ethnisch hergestellten Kategorie bedeutet demnach auch immer die Außerachtlassung anderer Kategorisierungsmöglichkeiten (Bergmann 2010, S. 158). Kategorisierungen, auch ethnisierte, sind somit kontextabhängig und verweisen auf Zusammenhänge: Das heißt, dass sie in ihrer Indexikalität, also relativ zu den Sprechenden und Handelnden und dem jeweiligen Kontext, betrachtet werden. Unter dem Stichwort der „situativen Angemessenheit“ beschreibt bereits Michael Moerman (1974) die Kategorisierungsmöglichkeit Ethnizität als Differenzierungsleistung und als Grenzziehung, die ohne Kontextualisierung nicht zu erschließen sei. „[U]sing one member of a set of identifications provides the context which makes other members of that set appropriate. Using the label ,Negro“ provides the context which makes labels like ,White' or ,Mexican appropriate" (ebd., S. 62). Demnach gilt es zu fragen, unter welchen Bedingungen und in welchen Kontexten ethnisierte Zugehörigkeiten und Grenzziehungen stabilisieren oder destabilisieren und dabei für unterschiedliche Akteur"innen nutzbar gemacht werden.

„Es reicht nicht, dass eine Kategorisierung stattfindet [...], entscheidend ist vielmehr, ob in sozialen Prozessen - in Interaktionen, Biografien, Verfahren, Moden, Diskursen usw. an diesen Anknüpfungspunkt angeschlossen wird, ob es also zur Wiederaufnahme einer Unterscheidung in deren Verlauf kommt, so dass ihre soziale Relevanz aufgebaut wird“ (Hirschauer 2014, S. 183).

Kategorisierung ist also darüber hinaus immer an Aushandlungen dieser Kategorien geknüpft, die empirisch und analytisch gefasst werden müssen bzw. in dieser Aushandlung erst empirisch beobachtbar werden. Eine besondere Stabilisierung ethnisierter Grenzziehungen findet sich in der politisch-territorialen Grenze, die sowohl als „Institution wie auch als Anordnung sozialer Beziehungen“ (Mezzadra/Neilson 2008) aus einem komplexen Zusammenspiel verschiedener sozialer und institutionalisierter Grenzziehungen entsteht. Sandro Mezzadra und Brett Neilson (2008) beschreiben das Zusammenwirken von Ethnisierung und (sozialen) Grenzen als in ständiger Auseinandersetzung begriffen und heben damit die diesem Spannungsfeld innewohnende Dynamik und Veränderbarkeit hervor. Im Wechselverhältnis zwischen institutionalisierten Zuschreibungen und der andauernden Infragestellung derselben finden daher ständige diskursive Verschiebungen sozialer Grenzen und erneute Grenzziehungen statt.

Diesen theoretisch-konzeptionellen Überlegungen wird im Folgenden anhand einer empirisch beobachtbaren Ausformung, nämlich der Nation, und ihrem Wechselverhältnis zu politischterritorialen Grenzen nachgespürt, da staatlich-ethnisierte Grenzziehungspraktiken als eine der, vielleicht als die prägnanteste Ausformung der oben beschriebenen Prozesse gelesen werden können, in denen sich Praktiken der Zuschreibung von Differenz stabilisieren und institutionalisieren. 


\section{Border(s), Ethnizität, Nation}

In vielerlei Hinsicht kann die Nation, insbesondere der Nationalstaat, als ein mögliches Ziel von Ethnisierungsprozessen gesehen werden: Als der Fall, in dem ethnisierende Narrative einer gemeinsamen Geschichte, Kultur und Sprache eine institutionalisierte Entsprechung auf politischer (und ökonomischer) Ebene gefunden haben. Es soll zunächst der Frage nachgegangen werden, in welchem Verhältnis Ethnisierungs- und Nationalisierungsprozesse stehen, wobei neben der sozialen boundary hier die territoriale border besonders wirkmächtig wird. Anschließend wird es um die territoriale Grenze von Nationen bzw. Nationalstaaten und ihre historische Kontingenz gehen. Drittens werden an nationalstaatlichen Grenzen Ein- und Ausschlüsse, Mitgliedschaften sowie Passierregeln (rules for passing) anders institutionalisiert als in sich ,nur' ethnisch verstehenden Gruppen.

\subsection{Zum Verhältnis von Ethnisierungsprozessen und Nation}

Es scheint heutzutage so gut wie unmöglich, von (De-)Ethnisierungsprozessen zu sprechen, ohne den Nationalstaat als Relation mit in den Blick zu nehmen. Die Aufteilung des verfügbaren Territoriums in (National-)Staaten bringt es mit sich, dass die Aushandlung ethnisierter Einund Ausschlüsse gegenwärtig immer in Auseinandersetzung mit mindestens einem (nationalen) Staat stattfindet. Dies gilt auch für Prozesse, in denen vormals als ethnisch wahrgenommene Grenzen an Relevanz verlieren, verschwimmen oder gänzlich in der staatlich bestimmten Zugehörigkeitskategorie aufgehen. Die Beziehung zwischen Nationalstaat und Ethnisierungsprozessen ist auch aufgrund der - nicht nur (national)staatlichen - politischen Steuerungsversuche dieser Prozesse so stark (vgl. Alonso 1994; Brubaker 2002; Bös 2007).

Wie Ethnisierungsprozesse generell beruhen auch Nationalisierungsprozesse auf Ein- und Ausschlüssen, die zumeist anhand von (zugeschriebener) Herkunft, Sprache, Kultur oder auch Religion legitimiert werden (vgl. Alonso 1994; Suny 2001). Wie verhalten sich Ethnisierungsund Nationalisierungsprozesse aus grenztheoretischer Perspektive zueinander? Ist der Unterschied lediglich darin zu sehen, dass der Nationalstaat durch eine stärkere Institutionalisierung die nationale Zugehörigkeit leichter stabilisieren kann - beispielsweise durch (erzwungene) sprachliche und kulturelle Homogenisierung (vgl. Weber 1976)? Einige Stimmen (vgl. Jenkins 1994; Wimmer 2008a; 2013) sehen in Ethnizität die relevantere Kategorie. Nation - wie auch Rasse - sei ein historisch spezieller Fall von Ethnisierungsprozessen. Brubaker stimmt $\mathrm{zu}$, dass es sich bei beiden Konzepten um „a single integrated family of forms of cultural understanding, social organization, and political contestation" (Brubaker 2009, S. 22, Herv. i. O.) handele, argumentiert aber darüber hinaus, dass in dem gegenwärtig andauernden historischen Kontext Ethnizität und Nation unterschiedliche Ansprüche erheben: „In a world in which polities are legitimated only by claims to nationhood, the category nation does different sorts of organizational work than the categories race and ethnicity“ (Brubaker 2014, S. 805). Wer Anspruch auf den Status einer Nation erhebt, verlangt demnach heute auch nach (größerer) politischer Unabhängigkeit, die Anerkennung als ethnische Gruppe würde hingegen eher auf den Anspruch spezieller Minderheitenrechte abzielen. Ethnische Regionen und ethnische Minderheiten können dieser Konzeptualisierung nach Teil von größeren (national)staatlichen Gebilden sein, sich als eigenständige Nationen verstehende Minderheitenkollektive nicht in 
demselben Maße. Ana María Alonso (1994, S. 391) verweist darauf, dass Ethnisierungsprozesse als das partikularisierende Gegenstück zu den homogenisierenden Dynamiken der Nationalstaatsbildung zu verstehen seien, wobei eine Hierarchie entstünde, welche ethnisierte Gruppen der nationalen Mehrheit unterordne. Gleichgültig ob ein sich als Nation verstehendes Kollektiv im postsowjetischen Raum nach größerer politischer Souveränität oder Unabhängigkeit strebt (z.B. Abchasien und Südossetien in Georgien oder Transnistrien in der Republik Moldau), ob es um de- und postkoloniale Nationenbildung geht oder ob die Auseinandersetzungen sich im zeitgenössischen Westeuropa abspielen (z.B. in Katalonien, dem Baskenland oder Schottland): Politische Akteur*innen können nationale Narrative entwickeln bzw. sich auf diese berufen, deren Anspruch heute international weithin verstanden wird (vgl. Hobsbawm/Ranger 1983; Bhabha 1990; Alonso 1994; Suny 2001; Brubaker 2009; vgl. auch Lehner in diesem Band). ${ }^{4}$ Des Weiteren folgen die Mitgliedschaftskriterien staatlichen anstelle von rein ethnonationalen Erwägungen (Brubaker 2014, S. 806).

Die Bedeutung politischer Souveränität für die Vorstellung nationaler Kollektive findet sich bereits in der Idee der Imagined Communities bei Benedict Anderson (1983). Wichtig ist hier nicht nur die Möglichkeit, an politischen Entscheidungsprozessen teilzuhaben, sondern auch den als national bestimmten Raum zunächst geografisch einzugrenzen und dann zu kontrollieren. Der Nationalstaat bezieht sich mithin im Vergleich zur ,ethnischen Gruppe' auch anders, nämlich exklusiv, auf das als ,national‘ vorgestellte Territorium. Die Leave-Kampagne zum Brexit-Referendum 2016 zeigt beispielhaft, dass die Wirkmächtigkeit dieser Vorstellung unabhängig von ihrer empirischen Umsetzbarkeit funktioniert. Unter dem Slogan Take back control wurde mit dem Brexit nicht nur ,vollkommene‘ politische Souveränität gegenüber der Europäischen Union (EU) verlangt, sondern auch die Kontrolle über die Grenze und damit die europäische Arbeitsmigration nach Großbritannien.

Ein territorialer Bezug ist allerdings auch in vielen ethnisierenden Vorstellungen vorhanden (vgl. Alonso 1994; Bös 2007). Räumliche Grenzziehungen können hierbei in ihrer (Un-)Schärfe stark variieren. Dies gilt einerseits in Bezug auf die vorgestellte Ausdehnung des proklamierten ,Ursprungsterritoriums', andererseits sorgt die Herausbildung komplexer und transnationaler ethnoscapes im Zuge der fortschreitenden Globalisierung (Appadurai 1996; zur Transnationalität vgl. auch Vertovec 2009) und der mit ihr einhergehenden Superdiversität (vgl. Vertovec 2007; Blommaert 2013) für Räume, die den homogenisierenden Anspruch nationalisierender Vorstellungen unterlaufen. In diesen superdiversen ethnoscapes bekommt das alltägliche Zusammenleben größeres Gewicht (vgl. Padilla et al. 2015) bzw. werden lokale Zugehörigkeiten hergestellt, die nicht entlang ethnisierender Grenzen strukturiert sind (Vijver et al. 2015). Auch diese Zusammenhänge sind jedoch komplexer Natur: So beschreibt Katharine Tyler (2017) das Suburban Paradox of Conviviality and Racism in Postcolonial Britain, das einerseits von vertrauensvollen lokalen Beziehungen und Freundschaften über ethnisierte Grenzen hinweg geprägt ist, was aber andererseits nicht zu generell größerer Offenheit oder abnehmendem Alltagsrassismus führe.

4 Sind historische Bezüge recht regelmäßig ein wichtiger Bestandteil der Herstellung ethnischer und nationaler Zugehörigkeiten (Anderson 1983; Hobsbawm/Ranger 1983), sind sie für de- und postkoloniale Nationalisierungsprozesse so gut wie unverzichtbar (Ranger 1983; Suny 2001; Mamdani 2012). 


\subsection{Nation und border}

Der Nationalstaat bezieht sich also - zumindest dem Anspruch nach - exklusiv und kontrollierend auf das nationale Territorium und seine Grenzen, was ihn von Ethnisierungsprozessen unterscheidet und gleichzeitig für politische Akteur*innen eben dieser Ethnisierungsprozesse zum angestrebten Ziel machen kann (vgl. Wimmer/Min 2006; Brubaker 2014). Aus der Perspektive einer grenztheoretischen Ethnisierungsanalyse stellen sich zwei zentrale Fragen an die vorgestellte Eins-zu-Eins-Relation zwischen Nation und Territorium, welche Anssi Paasi als „deeply territorial ideology” (2016, S. 23) diagnostiziert. Die vielleicht offensichtlichste ist die Frage danach, wie mit komplexeren räumlichen Gefügen umgegangen wird: Was also passiert, wenn Ethnisierungsprozesse nicht in die jeweils aktuellen territorialen - z.B. kolonial gezogenen - staatlichen Grenzen passen? Die zweite Frage dreht sich um soziale Aspekte: einerseits um die Herstellung und Kontrolle von Mitgliedschaft und Zugehörigkeit sowie andererseits um die Herstellung der nationalen Grenze als einer ebensolchen beim Grenzübertritt selbst (vgl. 3.3).

$\mathrm{Zu}$ Kolonialzeiten gezogene Grenzen bieten sich besonders zur Beantwortung der ersten Frage an: An ihnen lässt sich die Kontextabhängigkeit politisch-territorialer Grenzen und die ,Gemachtheit‘ sowohl dieses (national)staatlichen Raumes als auch Zugehörigkeit stiftender Symbole und Narrative besonders deutlich nachzeichnen. Hierbei sei angemerkt, dass kollektivierende Homogenisierungen - offenkundig z.B. an Auseinandersetzungen um (meist nationale) Standardsprachen abzulesen (vgl. Joseph 2004; Ayres 2009) - in den seltensten Fällen gewaltfrei ablaufen (für Frankreich vgl. Weber 1976). Der europäische Kolonialismus ist auch deswegen aus grenztheoretischer Perspektive so interessant, weil in den Kolonien anhand von Kartografie und ethnisierenden bzw. rassifizierenden Kategorisierungen Wissens- und Kontrollformen herausgebildet wurden, die heute zum Instrumentarium (national)staatlichen Ordnungsstrebens und seiner Grenzregime gehören (Anderson 1983; Appadurai 1996). Neu am europäischen Kolonialismus ist allerdings nicht die Kategorisierung seiner Subjekte per se, beispielsweise zu Steuerzwecken anhand religiöser Zugehörigkeiten (vgl. für das Osmanische Reich Barkey 2008). Neu sind jedoch das Bestreben, die teils neu hergestellten, vermeintlich eindeutigen rassifizierenden Kategorien auf sämtliche Subjekte (auch Frauen und Kinder) anzuwenden und festzuschreiben, diese mit einem bestimmten Territorium in Verbindung zu bringen (vgl. Anderson 1983; Alonso 1994; Garuba 2002; Mamdani 2012), sowie der orientalisierende Blick auf die derart kategorisierten kolonialen Subjekte (vgl. Said 1978; Appadurai 1996).

Diese Entwicklung und Festschreibung ethnonationaler Kategorien sowie ihre räumlichen Verortungen wirken bis heute auf zweierlei Weise fort. Zum einen wurden und werden koloniale Kategorisierungen regelmäßig für dekoloniale Freiheitsbewegungen übernommen und relevant gemacht (vgl. Anderson 1983; Slezkine 1994; Appadurai 1996; Mamdani 2012; Gordon 2018). Eine Vielzahl von Autor*innen warnt in diesem Zusammenhang davor, die präkolonialen sozialen und politischen Gefüge als ,natürlich` oder ,zeitlos' zu essenzialisieren (vgl. Ranger 1983; Appadurai 1996; Bley 2005; Mamdani 2012). Die überwiegend eher zufällig gezogenen territorialen kolonialen Grenzen bieten - neben dem unsäglichen kolonialen Unrecht - zum anderen einer nationalisierten Welt nachvollziehbare Gründe für die Ethnisierung von Konflikten bzw. dem Streben nach nationaler politischer Souveränität in ebendiesen territorialen Grenzen. Wo nationale Vorstellungen und (postkoloniale) staatliche Grenzen sich nicht entsprechen, bil- 
den nationale Narrative, staatliche Grenzen überschreitende Verbindungen und Vorstellungen sowie staatliche Ordnungsversuche besonders sichtbare komplexe (und häufig gewaltvolle) Gefüge, welche ,einfache` und eindeutige Relationen von Territorium, Nation und Staat in Frage stellen. Als besonders markantes Beispiel seien hier kurdische nationalisierende Projekte genannt (vgl. Bruinessen 2000; Galip 2015).

\subsection{Verortung und Hervorbringung der nationalen Grenze}

In der herkömmlichen Vorstellung treffen Nationalstaaten genau an der territorialen Grenzlinie aufeinander, die Grenze wäre damit leicht zu bestimmen und zu verorten. Diese „gefeierte Linie“ (Elze 2015) stellt sich bei näherer Betrachtung jedoch als deutlich vielschichtiger und uneindeutiger dar. Bereits angesprochen wurden transnationale und potenziell superdiverse ethnoscapes, die das Streben nach nationaler Eindeutigkeit unterlaufen (vgl. Appadurai 1996; Vertovec 2007; 2009; Blommaert 2013; vgl. auch Schäfer in diesem Band). Konzeptionalisierungen wie nationale Grenzen überschreitende soziokulturelle Mitgliedschaftsräume (Rokkan 2000) oder das Addieren sprachlicher, religiöser, kultureller, politischer und ökonomischer Grenzebenen (vgl. Haselsberger 2014) öffnen den Blick für eine räumlich ausgedehntere und vielschichtigere Grenzregion (siehe auch Klatt in diesem Band). Deutlich kämpferischer verorten etwa Gloria Anzaldúa oder Chiara Brambilla die alltäglichen - auch politischen Auseinandersetzungen um Aus- und Einschlüsse in den geografisch umfassenderen und schwer einzugrenzenden borderlands (Anzaldúa 1987) bzw. borderscapes (Brambilla 2015). Dabei verlagert sich der analytische Blick von statischen Vorstellungen hin zu einer Untersuchung der Prozesse, in denen Grenzen hervorgebracht, verhandelt und unterwandert werden.

Wie bereits erwähnt, wird die (national)staatliche Mitgliedschaft anders organisiert als die in sich als ethnisch verstehenden Kollektiven (vgl. Brubaker 2014). Zur Mitgliedschaft qua Geburt gesellen sich institutionalisierte, rechtlich festgelegte Möglichkeiten, die Staatsbürgerschaft zu erlangen. Anhand der Kriterien zur Einbürgerung, also der Anerkennung der (national)staatlichen Mitgliedschaft, lässt sich auch ablesen, wie die nationale Kategorie jeweils verstanden wird. So ist Israel vermutlich der einzige Staat, der die Staatsbürgerschaft allein anhand der Religionszugehörigkeit verleiht. Nationalstaatlich institutionalisierte soziale Einschlüsse in Form der Staatsbürgerschaft bringen allerdings nicht notwendigerweise auch die allägliche Anerkennung der derart kategorisierten Personen mit sich. So gibt es in Deutschland beispielsweise einen rassifizierenden Diskurs um sogenannte ,Passdeutsche', der diese institutionalisierte Mitgliedschaft in Frage stellt (Vassiliou-Enz et al. 2017; Hoffmann 2020). Ein weiteres Beispiel für umkämpfte und hierarchisierte Zugehörigkeiten sind die ,koethnischen Migrationen aus dem postsowjetischen Raum nach Deutschland, Griechenland und Israel (vgl. Beiträge in Čapo Žmegač et al. 2010; Zeveleva 2017; Panagiotidis 2019).

Die auch an nationalstaatlichen Grenzen vorgenommenen Kontrollen der nationalen Mitgliedschaft sind Teil des borderwork (Rumford 2008) bzw. Grenzregimes (Hess/Tsianos 2007), die über Ein- oder Ausschluss bzw. den Grenzübertritt entscheiden. In diesen Grenzprozessen fallen mithin die politisch-territoriale border mit der soziokulturellen boundary zusammen und lassen sich gemeinsam untersuchen. In der in Dokumenten festgehaltenen Staatsbürgerschaft lässt sich eine Hierarchisierung derselben feststellen: Eine kleine Minderheit kann sich weltweit weitestgehend ungehindert über nationalstaatliche Grenzen hinweg bewegen und sich 
an anderen als dem Geburtsort aufhalten, der Mehrheit der Menschen bleibt dies versagt (besonders eindrücklich: Khosravi 2010). Illegalisierte Migrant*innen erfahren diesen Ausschluss unmittelbar und mit oft tödlicher Härte (vgl. Khosravi 2010; Fassin 2011). Die Kontrolle der nationalen Mitgliedschaft erstreckt sich gerade im Falle der illegalisierten Migration in Ländern des Globalen Nordens durch staatliche Strategien wie beispielsweise das Britische Hostile Environment ${ }^{5}$ inzwischen auf das gesamte Staatsgebiet (vgl. Rumford 2012; Cassidy et al. 2018).

\section{Ethnizität zwischen border und boundary: der Fall COVID-19}

Am Beispiel der COVID-19-Pandemie lässt sich das Zusammenspiel von Ethnisierungspraktiken und (nationalstaatlichen) Grenzziehungen sowie deren Institutionalisierung aktuell eindrücklich zeigen. Schnell kam es zu Grenzschließungen auch innerhalb des europäischen Schengenraumes, der nun nicht mehr ,nur' nach außen abgeschottet, sondern auch nach innen in seine einzelstaatlichen Teile zergliedert wurde (vgl. Wille/Kanesu 2020). Gleichzeitig bzw. bereits vor den Grenzschließungen kam es vermehrt zu rassistischen Angriffen auf als ,chinesisch` kategorisierte Personen, unabhängig von ihrer tatsächlichen Staatsbürgerschaft (vgl. Devakumar et al. 2020). Während im Juni 2020 die einschränkenden Maßnahmen in der EU mehr oder weniger langsam gelockert wurden und (national)staatliche Grenzen innerhalb des Schengenraumes wieder gemäß der früheren (ungleichen) Passierregeln überquert werden können, lassen sich bezeichnende ethnisierende Grenzziehungen feststellen. Diese greifen bestimmte Bevölkerungsgruppen als ,Problemgruppen' heraus, denen zugeschrieben wird, sich den Maßnahmen zur Eindämmung der Pandemie zu widersetzen oder diese nicht genügend umzusetzen. Standen zu Beginn der Pandemie häufig Glaubensgemeinschaften per se im Verdacht, in ihren Zusammenkünften dem Virus die Verbreitung zu ermöglichen, werden nun vermehrt Muslim*innen bzw. ethnisierte Minderheiten als besonders problematisch bewertet. ${ }^{6}$ Diese Bewertung wird mit einer vermeintlichen ,kulturellen Differenz' erklärt, womit in Europa bereits einschlägig verfestigte und institutionalisierte soziale Grenzziehungen abgerufen werden können (vgl. Sayyid 2018; Bayrakali/Hafez 2019). Ein Teil der osteuropäischen Arbeitsmigrant*innen in Deutschland wird auf andere Weise auf ihre Nationalität zurückgeworfen und diese problematisiert. Im Niedriglohnsektor sind diese Arbeitsmigrant" innen gezwungen, unter teils menschenunwürdigen Bedingungen zu leben und zu arbeiten. Die wenig überraschenden COVID-19-Ausbrüche werden sowohl in den behördlichen Kontrollmaßnahmen als auch in der Berichterstattung und gesellschaftlichen Debatte jedoch nicht nur auf die menschenunwürdigen Umstände zurückgeführt, sondern es wird immer wieder in ,nationalen“ Kategorien argumentiert. $^{7}$

Institutionalisierte Kontrollmaßnahmen zur Eindämmung der Pandemie sowie die Gesundheitsvorsorge sind nach wie vor auch in Europa größtenteils (national)staatlich organisiert. Da die Schwere der Pandemie offenbar sehr deutlich von den getroffenen Maßnahmen abhängt, scheint das Virus sich zumindest teilweise doch , an nationale Grenzen zu halten'. Gleichzeitig

5 Vermieter*innen und Arbeitgeber*innen sind im Rahmen des Hostile Environment gesetzlich dazu verpflichtet, den Aufenthaltsstatus ihrer potenziellen Mieter*innen bzw. Angestellten zu überprüfen und handeln sich bei Zuwiderhandlung empfindliche Geldstrafen ein.

6 So z.B. bei lokalen Ausbrüchen in Göttingen (Henning/Hilbert 2020) oder in Leicester (Mahamdallie 2020).

7 Beispielsweise wenn Nordrhein-Westfalens Ministerpräsident Armin Laschet vom „Bulgarenvirus“ spricht (https:/ /twitter.com/nicolediekmann/status/1273257798307061762). 
sind sowohl Angehörige ethnischer Minderheiten als auch Einkommensschwache besonders von COVID-19 betroffen ${ }^{8}$ (vgl. Ahmed et al. 2020; Pareek et al. 2020). Wie am Beispiel osteuropäischer Arbeitsmigrant*innen in Deutschland - oder auch der Arbeiter*innen in den Textilfabriken in Leicester - deutlich wird, sind diese häufig nicht ,nur' arm, ,nur' der jeweiligen Behördensprache nicht unbedingt mächtig oder ,nur' Angehörige ethnisierter bzw. nationaler Minderheiten. Dies befördert eine intersektionale Perspektive auf die von COVID-19 ausgelösten Grenzziehungsprozesse.

\section{Fazit und Ausblick}

Unser Ziel mit diesem Beitrag ist es, einerseits die komplexe Dynamik von ethnisierten Grenzen sowohl in der wissenschaftlichen Analyse als auch im empirisch beobachtbaren Gegenstand zu zeigen. Die Anerkennung der ethnisierten Grenze als komplexes Phänomen aus historischen, sozialen, kulturellen, (macht)politischen und temporalen Gefügen lässt einen detaillierten Blick in die jeweiligen Akteur"innenperspektiven zu. Ethnisierungspraktiken sind demnach als Teil intersektionaler Zugehörigkeiten aber auch selbst als intersektionale Handlungen im Wechselspiel von Zuschreibungen zu verstehen. Aus grenztheoretischer Perspektive erlaubt es der Fokus auf ethnonationale Aushandlungen von Zugehörigkeiten, die soziale boundary mit der politisch-territorialen border gemeinsam in den Blick zu nehmen und diese beiden Forschungsfelder füreinander fruchtbar zu machen (vgl. Schiffauer et al. 2018; Gerst/Krämer 2019). In (national)staatlichen Grenzkontrollen lässt sich eine Zuspitzung und Institutionalisierung von Ethnisierungsprozessen ausmachen - mit der Einschränkung, dass Staatsbürgerschaft, und damit ein rechtlich gleichwertiger Einschluss in Rechte und Pflichten, in den meisten Fällen durch einen mehr oder weniger transparenten Einbürgerungsprozess erlangt werden kann. Grenzen bzw. Grenzziehungen als spezifische Form der Differenzsetzung gedacht, an denen beispielsweise Ein- und Ausschlüsse kontrolliert und geregelt werden, bieten sich also geradezu an, um in Bezug auf Vorstellungen ethnisierter Kollektive relevant gemacht zu werden (vgl. Moerman 1965; PuruShotam 1998; Bergmann 2010; Höfler, i.E.).

Ein allzu starker gegenseitiger Bezug von Ethnisierungs- bzw. Nationalisierungs- und Grenzprozessen aufeinander birgt allerdings die Gefahr, dass Grenzforscher*innen schnell nur noch solche Grenzen interessieren, die als ,ethnisch“ relevant gemacht werden können. Aus grenztheoretischer Perspektive büßten Grenzziehungsprozesse dadurch an Komplexität ein (vgl. Gerst et al. 2018b) und die Multiplizität von Perspektiven auf die jeweilige Grenze sowie ihre unterschiedliche Relevanz für unterschiedliche Akteur*innen ginge analytisch verloren (vgl. Rumford 2012; Brambilla 2015). Eine andere Gefahr besteht darin, dass Ethnizität plötzlich nur noch aus Grenzziehungen zu bestehen scheint und beispielsweise die Herstellung von Gemeinsamkeiten, z.B. über geteilte Praktiken und Narrative, aus dem Blick gerät (vgl. PfaffCzarnecka 2011; Vallentin 2019). Dies geschieht besonders dann, wenn übersehen wird, dass Grenzprozesse auch Relationierungsprozesse sind, dass also Gruppen hergestellt werden, die zum einen miteinander in Beziehung gesetzt werden und die zum anderen homogenisieren, was zu ,beiden Seiten` der Grenze sortiert wird (vgl. Bowker/Star 2000; Tilly 2004; Karafillidis 2009; Hirschauer 2014). Auch dort, wo sich ethnisierte Grenzziehungen ausmachen lassen, sind längst nicht alle relevanten sozialen Grenzen ,ethnischer Natur'. Der enge Fokus auf eth-

8 In Großbritannien beispielsweise ist ihr Anteil unter den Todesfällen der Angestellten im Gesundheitswesen überproportional hoch (Marsh/McIntyre 2020; Public Health England 2020). 
nisierte Grenzziehungen kann dazu führen, die ethnisierte Grenze zu verabsolutieren, anstatt sie als einen spezifischen Fall der (sozialen) Grenze zu betrachten und in ihrem Verhältnis zu anderen Formen der Grenzziehung zu untersuchen (vgl. Hirschauer 2014). Setzt man eine soziale Grenze wie z.B. Ethnizität voraus, auch wenn man diese als eine von mehrfachen Zugehörigkeiten betrachtet, ist der Moment, in dem diese Differenz nicht relevant gemacht wird, also ,ruht', ,verblasst' oder ,verwischt', nur schwer zu beobachten (vgl. ebd.). Grenz- oder Ethnizitätsforscher*innen forschen dann an den sie interessierenden Lebenswirklichkeiten, Dynamiken oder Prozessen, vorbei‘. Werden Vorstellungen von Ethnizität stärker über Prozesse der Grenzziehung untersucht, können sowohl die Herstellung, Stabilisierung und Beständigkeit als auch die Überwindung und Auflösung solcher Grenzen in den Blick geraten. Dies macht wiederum die Beschreibung von Mehrfachzugehörigkeiten und das Zusammenwirken heterogener Differenzkategorien möglich (vgl. Anzaldúa 1987; Winker/Degele 2009; Hirschauer 2014).

Spricht man über die Hervorbringung ethnisierter Grenzen, muss schließlich auch über das unmaking oder undoing dieser Prozesse gesprochen werden (vgl. Bergmann 2010; Hirschauer 2014, S. 183). In Interaktionen lässt sich beispielsweise nachzeichnen, wie Teilnehmende daran ,scheitern', sich - z.B. in Auseinandersetzung mit staatlichen Institutionen - in ethnisierenden Kategoriensystemen einzuordnen (vgl. Vallentin 2019). Genauso kann die Betrachtung längerer Zeiträume für die Grenz- und Ethnizitätsforschung von Interesse sein. Beispielsweise werden in den USA Ir*innen, Italiener*innen und Jüd*innen heute als, Weiß`, gelesen', während sie zu Beginn des letzten Jahrhunderts noch als ,Schwarz' kategorisiert wurden (vgl. Wimmer 2008). Die angesprochene prozessorientierte Perspektive bringt es auch mit sich, ethnisierte Kollektive - gleichgültig ob sie im Nationalstaat organisiert sind oder dies auf größeren oder kleinteiligeren Ebenen geschieht - nicht als endgültig zu verabsolutieren. Das jeweils interessierende historische Moment ist dabei als ein in seiner Zeit eingebettetes Gefüge zu verstehen, in dem sich auch Spuren früherer Grenzziehungen finden lassen (vgl. Green 2009).

Zusammenfassend können wir festhalten, dass Ethnisierungsprozesse und soziokulturelle Grenzen also mehr oder weniger sichtbarer Gegenstand ständiger Aushandlungen über Einund Ausschlüsse sind, die z.B. in institutionalisierten Praktiken wie der Passvergabe und -kontrolle eine Verfestigung finden können. Trotz - oder gerade wegen - dieser ständigen Veränderungen liegt der Schluss nahe, dass Akteur*innen dazu tendieren, ethnonationale Grenzen letztlich biologistisch zu essenzialisieren, wenn sie diese relevant machen. Gerade deshalb ist es für Grenzforscher*innen von zentraler Bedeutung, diesen möglichen Essenzialisierungen nachzuspüren und den Akteur*innen in ihren jeweiligen Praxisfeldern und Umwelten zu folgen - auch wenn sich der Forschungsgegenstand über mehrere soziale und individuelle Felder erstreckt (vgl. zur multi-sited ethnography Marcus 1995).

\section{Weiterführende Literatur}

Barth, Fredrik (1969): Introduction. In: Ders. (Hrsg.): Ethnic Groups and Boundaries. The social organization of cultural differences. Oslo: Universitetsforlaget, S. 11-38.

Hirschauer, Stefan (2014): Un/doing Differences. Die Kontingenz sozialer Zugehörigkeiten. In: Zeitschrift für Soziologie 43, H. 3, S. 170-191.

Lamont, Michèle/Molnár, Virág (2002): The Study of Boundaries in the Social Sciences. In: Annual Review of Sociology 28, S. 167-195.

Römhild, Regina (2007): Fremdzuschreibungen - Selbstpositionierungen. Die Praxis der Ethnisierung im Alltag der Einwanderungsgesellschaft. In: Schmidt-Lauber, Brigitta (Hrsg.): Ethnizität und Migration. Einführung in Wissenschaft und Arbeitsfelder. Berlin: Dietrich Reimer, S. 157-177. 
Wimmer, Andreas (2008): The making and unmaking of ethnic boundaries: A multi-level process theory. In: American Journal of Sociology 113, H. 4, S. 970-1022.

\section{Literaturverzeichnis}

Ahmed, Faheem/Ahmed, Na'eem/Pissarides, Christopher/Stiglitz, Joseph (2020): Why inequality could spread COVID-19. In: The Lancet 5, H. 5, E240. DOI: 10.1016/S2468-2667(20)30085-2.

Alonso, Ana María (1994): The politics of space, time and substance: State formation, nationalism, and ethnicity. In: Annual Review of Anthropology 23, H. 1, S. 379-405.

Anderson, Benedict (1983): Imagined Communities. London: Verso.

Anzaldúa, Gloria (1987): Borderlands/La Frontera: The New Mestiza. San Francisco: aunt lute.

Appadurai, Arjun (1996): Modernity at Large: Cultural Dimensions of Globalization. Minneapolis: University of Minnesota Press.

Ayres, Alyssa (2009): Speaking Like a State: Language and Nationalism in Pakistan. Cambridge: Cambridge University Press.

Barkey, Karen (2008): Empire of Difference. The Ottomans in Comparative Perspective. Cambridge: Cambridge University Press.

Barth, Fredrik (1969): Introduction. In: Ders. (Hrsg.): Ethnic Groups and Boundaries. The social organization of cultural differences. Oslo: Universitetsforlaget, S. 11-38.

Barth, Fredrik (2000): Boundaries and connections. In: Cohen, Anthony P. (Hrsg.): Signifying Identities. Anthropological perspectives on boundaries and contested values. London/New York: Routledge, S. 1736.

Bayrakli, Enes/Hafez, Farid (Hrsg.) (2019): European Islamophobia Report 2018. Ankara/Istanbul/Washington D.C./Berlin/Brüssel: SETA I Foundation for Political, Economic and Social Research. www.seta v.org/en/assets/uploads/2019/09/EIR_2018.pdf, 17.8.2020.

Bergmann, Jörg (2010): Die kategoriale Herstellung von Ethnizität - Ethnomethodologische Überlegungen zur Ethnizitätsforschung. In: Müller, Marion/Zifonoun, Dariuš (Hrsg.): Ethnowissen. Soziologische Beiträge zu ethnischer Differenzierung und Migration. Wiesbaden: VS Verlag für Sozialwissenschaften, S. 155-169.

Bhabha, Homi K. (1990): DissemiNation: Time, narrative, and the margins of the modern nation. In: Ders. (Hrsg.): Nation and Narration. London: Routledge, S. 291-322.

Bley, Helmut (2005): Künstliche Grenze, natürliches Afrika? Um die Berliner Kongokonferenz von 1884 1885 ranken sich allerhand Mythen. In: Iz3w 282, S. 14-16.

Blommaert, Jan (2013): Ethnography, Superdiversity and Linguistic Landscapes. Bristol: Multilingual Matters.

Bös, Mathias (2005): Rasse und Ethnizität: Zur Problemgeschichte zweier Begriffe in der amerikanischen Soziologie. Wiesbaden: VS Verlag für Sozialwissenschaften.

Bös, Mathias (2007): Ethnizität und Grenzen in Europa. In: Deger, Petra/Hettlage, Robert (Hrsg.): Der europäische Raum: Die Konstruktion europäischer Grenzen. Wiesbaden: VS Verlag für Sozialwissenschaften, S. 49-69.

Bowker, Geoffrey C./Star, Susan Leigh (2000): Sorting things out. Classification and its consequences. Cambridge/London: MIT Press.

Brambilla, Chiara (2015): Exploring the critical potential of the borderscapes concept. In: Geopolitics 20, H. 1, S. 14-34.

Brubaker, Rogers (2002): Ethnicity without groups. In: Archives Europeénnes de Sociologie 43, H. 2, S. $163-189$.

Brubaker, Rogers (2004): Ethnicity without Groups. Cambridge: Harvard University Press.

Brubaker, Rogers (2007): Ethnizität ohne Gruppen. Hamburg: Hamburger Edition.

Brubaker, Rogers (2009): Ethnicity, race, and nationalism. In: Annual Review of Sociology 35, H. 1, S. 21-42.

Brubaker, Rogers (2014): Beyond ethnicity. In: Ethnic and Racial Studies 37, H. 5, S. 804-808.

Bruinessen, Martin van (2000): Transnational aspects of the Kurdish question. Working paper, Robert Schuman Centre for Advanced Studies, European University Institute, Florenz.

Cassidy, Kathryn/Yuval-Davis, Nira/Wemyss, Georgie (2018): Debordering and everyday (re)bordering in and of Dover: post-borderland borderscapes. In: Political Geography 66, S. 171-179.

Čapo Žmegač, Jasna/Voß, Christian/Roth, Klaus (Hrsg.) (2010): Co-Ethnic Migrations Compared. Central and Eastern European Contexts. München/Berlin: Otto Sagner.

Devakumar, Delan/Shannon, Gordon/Bhopal, Sunil S./Abubakar, Ibrahim (2020): Racism and discrimination in COVID-19 responses. In: The Lancet, Correspondence 395, H. 10231, S. 1194. 
Elze, Theresa (2015): Die gefeierte Linie. Rituale und Komplizenschaft an der US-mexikanischen Grenze. Bielefeld: transcript.

Fassin, Didier (2011): Policing borders, producing boundaries. The governmentality of immigration in dark times. In: Annual Review of Anthropology 40, S. 213-226.

Feischmidt, Margit (2007): Ethnizität - Perspektiven und Konzepte der ethnologischen Forschung. In: Schmidt-Lauber, Brigitta (Hrsg.): Ethnizität und Migration. Einführung in Wissenschaft und Arbeitsfelder. Berlin: Dietrich Reimer, S. 51-68.

Galip, Özlem Belçim (2015): Imagining Kurdistan: Identity, Culture and Society. London/New York: I.B. Tauris.

Garuba, Harry (2002): Mapping the Land/Body/Subject: Colonial and Postcolonial Geographies in African Narrative. In: Alternation 9, H. 1, S. 87-116.

Gerst, Dominik/Klessmann, Maria/Krämer, Hannes/Sienknecht, Mitja/Ulrich, Peter (2018a): Komplexe Grenzen. Perspektiven aktueller Grenzforschung. In: Berliner Debatte Initial 29, H. 1, S. 3-11.

Gerst, Dominik/Klessmann, Maria/Krämer, Hannes/Sienknecht, Mitja/Ulrich, Peter (Hrsg.) (2018b): Komplexe Grenzen. Themenheft im Journal Berliner Debatte Initial 29, H. 1.

Gerst, Dominik/Krämer, Hannes (2019): Die methodologische Fundierung kulturwissenschaftlicher Grenzforschung. In: Kleinmann, Sarah/Peselmann, Arnika/Spieker, Ira (Hrsg.): Kontaktzonen und Grenzregionen. Kulturwissenschaftliche Perspektiven. Leipzig: Leipziger Universitätsverlag, S. 47-70.

Gordon, Uri (2018): Anarchism and Nationalism: On the Subsidiarity of Deconstruction. In: Jun, Nathan (Hrsg.): Brill's Companion to Anarchism and Philosophy. Leiden: Brill, S. 196-215.

Green, Sarah (2009): Lines, traces and tidemarks: Reflections on forms of borderli-ness. Working paper, EastBordNet COST Action IS0803.

Galip, Özlem Belçim (2015): Imagining Kurdistan: Identity, Culture and Society. London/New York: I.B. Tauris.

Handler, Richard (1985): On Dialogue and Destructive Analysis: Problems in Narrating Nationalism and Ethnicity. In: Journal of Anthropological Research 41, H. 2, S. 171-182.

Haselsberger, Beatrix (2014): Decoding borders. Appreciating border impacts on space and people. In: Planning Theory \& Practice 15, H. 4, S. 505-526.

Henning, Philipp/Hilbert, Jörg (2020): Vorverurteilung nach Corona-Ausbruch? Panorama, 11.6.2020. www.daserste.ndr.de/panorama/archiv/2020/Goettingen-Vorverurteilung-nach-Corona-Ausbruch ,goettingen1264.html, 17.8.2020.

Hess, Sabine/Tsianos Vassilis (2007): Europeanizing transnationalism! Provinzializing Europe! Konturen eines neuen Grenzregimes. In: Transit Migration Forschungsgruppe (Hrsg.): Turbulente Ränder. Neue Perspektiven auf Migration an den Rändern Europas. Bielefeld: transcript, S. 23-38.

Hirschauer, Stefan (2014): Un/doing Differences. Die Kontingenz sozialer Zugehörigkeiten. In: Zeitschrift für Soziologie 43, H. 3, S. 170-191.

Hobsbawm Eric/Ranger, Terence (Hrsg.) (1983): The Invention of Tradition. Cambridge: Cambridge University Press.

Hoffmann, Ludger (2020): Zur Sprache des Rassismus. In: Sprachreport 36, H. 1, S. 40-47.

Höfler, Concha Maria (i.E.): Boundaries and Belonging in the Greek Community of Georgia. Baden-Baden: Nomos.

hooks, bell (1981): Ain't I a woman? Black women and feminism. Boston: South End Press.

Jenkins, Richard (1994): Rethinking Ethnicity: Identity, Categorization and Power. In: Ethnic and Racial Studies 17, S. 197-223.

Jenkins, Richard (2008): Rethinking Ethnicity: Arguments and Explorations. London/Thousand Oaks: Sage.

Joseph, John E. (2004): Language and Identity: National, Ethnic, Religious. Basingstoke: Palgrave Macmillan.

Karafillidis, Athanasios (2009): Entkopplung und Kopplung - wie die Netzwerktheorie zur Bestimmung sozialer Grenzen beitragen kann. In: Häußling, Roger (Hrsg.): Grenzen von Netzwerken. Wiesbaden: VS Verlag für Sozialwissenschaften, S. 105-131.

Khosravi, Shahram (2010): The ,illegal' traveller: An auto-ethnography of borders. Basingstoke/New York: Palgrave Macmillan.

Lamont, Michèle/Molnár, Virág (2002): The Study of Boundaries in the Social Sciences. In: Annual Review of Sociology 28, H. 1, S. 167-195.

Mahamdallie, Hassan (2020): Race, class and coronavirus: Lessons from Leicester. dream deferred, 2.7.2020. www.dreamdeferred.org.uk/2020/07/race-class-and-coronavirus-lessons-from-leicester/, 17.8.2020.

Mahmood, Cynthia Keppley (1996): Fighting for Faith and Nation: Dialogues with Sikh Militants. Philadelphia: University of Pennsylvania Press. 
Mamdani, Mahmood (2012): Define and Rule: Native as Political Identity. Cambridge/London: Harvard University Press.

Marcus, George (1995): Ethnography in/of the World System: The Emergence of Multi-Sited Ethnography. In: Annual Review of Anthropology 24, H. 1, S. 95-117.

Marsh, Sarah/McIntyre, Niamh (2020): Six in 10 UK health workers killed by Covid-19 are BAME. The Guardian, 25.5.2020. www.theguardian.com/world/2020/may/25/six-in-10-uk-health-workers-killed-by -covid-19-are-bame, 17.8.2020.

Mezzadra, Sandro/Neilson, Brett (2008): Die Grenze als Methode, oder die Vervielfältigung der Arbeit. www.transversal.at/transversal/0608/mezzadra-neilson/de, 23.5.2020.

Moerman, Michael (1965): Ethnic identification in a complex civilization: Who are the Lue? In: American Anthropologist 67, H. 5, S. 1215-1230.

Moerman, Michael (1974): Accomplishing Ethnicity. In: Turner, Roy. (Hrsg.): Ethnomethodology: Selected Readings. Harmondsworth: Penguin Education, S. 54-68.

Moya, Paula M. L./Hames-Garcia, Michael R. (Hrsg.) (2000): Reclaiming ldentity: Realist Theory and the Predicament of Postmodernism. Berkeley: University of California Press.

Müller, Marion/Zifonun, Dariuš (2010): Ethnowissen. Soziologische Beiträge zu ethnischer Differenzierung und Migration. Wiesbaden: VS Verlag für Sozialwissenschaften.

Nail, Thomas (2016): Theory of the Border. New York: Oxford University Press.

Newman, David (2006): Borders and Bordering. Towards an Interdisciplinary Dialogue. In: European Journal of Social Theory 9, H. 2, S. 171-186.

Orywal, Erwin/Hackstein, Katharina (1993): Ethnizität - die Konstruktion ethnischer Wirklichkeiten. In: Schweizer, Thomas/Schweizer, Margarete/Kokot, Waltraud (Hrsg.): Handbuch der Ethnologie. Berlin: Dietrich Reimer, S. 593-611.

Paasi, Anssi (2016): Dancing on the graves: Independence, hot/banal nationalism and the mobilization of memory. In: Political Geography 54, S. 21-31.

Padilla, Beatriz/Azevedo, Joana/Olmoz-Alcaraz, Antonia (2015): Superdiversity and conviviality: exploring frameworks for doing ethnography in Southern European intercultural cities. In: Ethnic and Racial Studies 38, H. 4, S. 621-635.

Panagiotidis, Jannis (2019): The unchosen ones. Diaspora, Nation, and Migration in Israel and Germany. Bloomington: Indiana University Press.

Pareek, Manish/Bangash, Mansoor N./Pareek, Nilesh/Pan, Daniel/Sze, Shirley/Minhas, Jatinder S./Hanif, Wasim/Khunti, Kamlesh (2020): Ethnicity and COVID-19: An urgent public health research priority. In: The Lancet 395, H. 10234, S. 1421-1422.

Parker, Noel/Vaughan-Williams, Nick (2012): Critical Border Studies: Broadening and Deepening the ,Lines in the Sand'Agenda. In: Geopolitics 17, H. 4, S. 727-733.

Pfaff-Czarnecka, Joanna (2011): From ,identity' to ,belonging' in social research: Plurality, social boundaries, and the politics of the self. In: Albiez, Sarah/Castro, Nelly/Jüssen, Lara/Youkhana, Eva (Hrsg.): Ethnicity, Citizenship and Belonging: Practices, Theory and Spatial Dimensions. Etnicidad, ciudanía y pertenencia: prácticas, teoría y dimensiones espaciales. Frankfurt/M./Madrid: Iberoamericana Vervuert, S. 199-219.

Public Health England (2020): Beyond the data: Understanding the impact of COVID-19 on BAME groups. www.assets.publishing.service.gov.uk/government/uploads/system/uploads/attachment_data/file/ 892376/COVID_stakeholder_engagement_synthesis_beyond_the_data.pdf, 17.8.2020.

PuruShotam, Nirmala (1998): Negotiating Language, Constructing Race. Disciplining Difference in Singapore. Berlin/New York: Mouton de Gruyter.

Ranger, Terence (1983): The invention of tradition in colonial Africa. In: Hobsbawm, Eric/Ranger, Terence (Hrsg.): The Invention of Tradition. Cambridge: Cambridge University Press, S. 211-262.

Römhild, Regina (2007): Fremdzuschreibungen - Selbstpositionierungen. Die Praxis der Ethnisierung im Alltag der Einwanderungsgesellschaft. In: Schmidt-Lauber, Brigitta (Hrsg.): Ethnizität und Migration. Einführung in Wissenschaft und Arbeitsfelder. Berlin: Dietrich Reimer, S. 157-177.

Rokkan, Stein (2000): Staat, Nation und Demokratie. Frankfurt/M.: Suhrkamp.

Rumford, Chris (2008): Introduction: Citizens and borderwork in Europe. In: Space and Polity 12, H. 1, S. $1-12$.

Rumford, Chris (2012): Towards a multiperspectival study of borders. In: Geopolitics 17, H. 4, S. 887902.

Said, Edward (1978): Orientalism. London: Penguin.

Sayyid, S. (2018): Islamophobia and the Europeanness of the other Europe. In: Patterns of Prejudice 52, H. 5, S. 420-435. 
Schiffauer, Werner/Koch, Jochen/Reckwitz, Andreas/Schoor, Kerstin/Krämer, Hannes (2018): Borders in Motion: Durabilität, Permeabilität, Liminalität. Working Paper B/ORDERS IN MOTION 1. DOI:10.11584/B-ORDERS. 1.

Simmel, Georg (1908/2013): Soziologie. Untersuchungen über die Formen der Vergesellschaftung. 7. Aufl., Berlin: Duncker \& Humblot.

Slezkine, Yuri (1994): The USSR as a communal apartment, or how a socialist state promoted ethnic particularism. In: Slavic Review 53, H. 2, S. 414-452.

Sökefeld, Martin (2007): Problematische Begriffe: „Ethnizität“, „Rasse“, „Kultur“, „Minderheit“. In: Schmidt- Lauber, Brigitta (Hrsg.): Ethnizität und Migration. Einführung in Wissenschaft und Arbeitsfelder. Berlin: Dietrich Reimer, S. 31-50.

Solleder, Stefan (2017): Die Visualisierung symbolischer Ordnungen im Kontext gewalttätiger Konflikte. Humboldt-Universität zu Berlin. DOI: 10.18452/18620.

Spivak, Gayatri Chakravorty (1988): Subaltern Studies. Deconstructing Historiography. In: Guha, Ranajit/Spivak, Gayatri Chakravorty (Hrsg.): Selected Subaltern Studies. Oxford: Oxford University Press, S. 3-44.

Suny, Ronald Grigor (2001): Constructing primordialism: Old histories for new nations. In: Journal of Modern History 73, H. 4, S. 862-896.

Tilly, Charles (2004): Social Boundary Mechanisms. In: Philosophy of the Social Sciences 34, H. 2, S. $211-$ 236.

Tyler, Katherine (2017): The suburban paradox of conviviality and racism in postcolonial Britain. In: Journal of Ethnic and Migration Studies 43, H. 11, S. 1890-1906.

Vallentin, Rita (2019): Belonging and Language Use. Narrating, categorizing and positioning in a Guatemalan highland community. Bern: Peter Lang.

Vassiliou-Enz, Konstantina/Lanzke, Alice/Bax, Daniel (2017): Am Anfang ist das Wort. In: Sprachreport 33 , H. 2, S. 28-35.

Vertovec, Steven (2007): Super-diversity and its implications. In: Ethnic and Racial Studies 30, H. 6, S. 1024-1054.

Vertovec, Steven (2009): Transnationalism. London/New York: Routledge.

Vijver, Fons J.R. van de/Blommaert, Jan/Gkoumasi, Georgia/Stogianni, Maria (2015): On the need to broaden the concept of ethnic identity. In: International Journal of Intercultural Relations 46, S. 36-46.

Voss, Christian (2018): Slawischsprachige Grenzminderheiten in Nordgriechenland. In: Berliner Debatte Initial 29, H. 1, S. 53-61.

Wille, Christian/Kanesu, Rebekka (Hrsg.) (2020): Bordering in pandemic times. Insights into the COVID-19 lockdown. UniGR-Center for Border Studies, Borders in Perspective, H. 4.

Wimmer, Andreas (2000): Ethnizität. In: Streck, Bernhard (Hrsg.): Wörterbuch der Ethnologie. Wuppertal: DuMont, S. 53-55.

Wimmer, Andreas (2008a): The making and unmaking of ethnic boundaries: a multi-level process theory. In: American Journal of Sociology 113, H. 4, S. 970-1022.

Wimmer, Andreas (2008b): Ethnische Grenzziehungen in der Immigrationsgesellschaft. Jenseits des Herder'schen Commonsense. In: Kalter, Frank (Hrsg.): Migration und Integration. Kölner Zeitschrift für Soziologie und Sozialpsychologie, H. 48, S. 57-80.

Wimmer, Andreas (2013): Ethnic Boundary Making. Institutions, Power, Networks. Oxford/New York: Oxford University Press.

Wimmer, Andreas/Min, Brian (2006): From empire to nation-state: Explaining wars in the modern world, 1816-2001. In: American Sociological Review 71, H. 6, S. 867-897.

Winker, Gabriele/Degele, Nina (2009): Intersektionalität. Zur Analyse sozialer Ungleichheiten. Bielefeld: transcript.

Weber, Eugen (1976): Peasants into Frenchmen. The Modernization of Rural France 1870-1914. Stanford: Stanford University Press.

West, Candace/Fenstermaker, Sarah B. (1995): Doing Difference. In: Gender \& Society 9, H. 1, S. 8-37.

Wolff, Stephan (2006): Textanalyse. In: Ayaß, Ruth/Bergmann, Jörg R. (Hrsg.): Qualitative Methoden in der Medienforschung. Reinbek: Rowohlt, S. 245-273.

Wolff, Stephan/Schönefeld, Daniel (2011): Der konversationsanalytische Zugang zur Interkulturalität. In: Bosse, Elke/Kreß, Beatrix/Schlickau, Stephan (Hrsg.): Methodische Vielfalt in der Erforschung interkultureller Kommunikation an deutschen Hochschulen. Frankfurt/M.: Peter Lang, S. 131-144.

Zeveleva, Olga (2017): States and standardisation: constructing the co-ethnic migrant story in Germany. In: Journal of Ethnic and Migration Studies 45, H. 4, S. 636-655. 